\title{
Motor Estimation using Heterogeneous Sets of Objects in Conformal Geometric Algebra
}

\author{
Lars Tingelstad* and Olav Egeland
}

\begin{abstract}
In this paper we present a novel method for nonlinear rigid body motion estimation from noisy data using heterogeneous sets of objects of the conformal model in geometric algebra. The rigid body motions are represented by motors. We employ state-of-the-art nonlinear optimization tools and compute gradients and Jacobian matrices using forward-mode automatic differentiation based on dual numbers. The use of automatic differentiation enables us to employ a wide range of cost functions in the estimation process. This includes cost functions for motor estimation using points, lines and planes. Moreover, we explain how these cost functions make it possible to use other geometric objects in the conformal model in the motor estimation process, e.g., spheres, circles and tangent vectors. Experimental results show that we are able to successfully estimate rigid body motions from synthetic datasets of heterogeneous sets of conformal objects including a combination of points, lines and planes.
\end{abstract}

Mathematics Subject Classification. Primary 15A66; Secondary 00A00.

Keywords. Rigid body motion estimation, Geometric algebra, Automatic differentiation, Optimization.

\section{Introduction}

Rigid body motion or pose estimation in geometric algebra has been an active research topic over the last decades, and it has been investigated using the conformal model since its introduction in 2001 by Li, Hestenes and Rockwood [15]. Lasenby et al. [14] presented attitude estimation using rotors in the 3D Euclidean model. Daniilidis [5] presented a solution to the handeye calibration problem in robotics using dual quaternions. This solution was later extended to the motor algebra in [2], and to the conformal model in [18]. Rosenhahn and Sommer [19] presented 2D-3D pose estimation using stratification of spaces. Further, Gebken, Perwass and Sommer [9] presented methods for $2 \mathrm{D}-3 \mathrm{D}$ and $3 \mathrm{D}-3 \mathrm{D}$ rigid body motion estimation from point data

${ }^{*}$ Corresponding author. 
in the conformal model using a tensor implementation for the numerical computations.

Most estimation techniques based on geometric algebra employ singular value decomposition or other linear least-squares methods. In contrast to this, Valkenburg and Alwesh [25] employed non-linear optimization in a calibration method of multiple stationary 3D points as part of an optical positioning system using the conformal model of geometric algebra. Perwass [17] used non-linear optimization in 3D-reconstruction from multiple view geometry in the projective model of geometric algebra. The methods of $[17,25]$ made use of multivector differentiation in geometric calculus [12] to compute gradients and Jacobian matrices.

In [24] we presented methods and implementation details for automatic differentiation $[13,22]$ of multivector valued functions, which were used for computing gradients and Jacobian matrices in nonlinear optimization. Both matrix and multivector implementations were presented. Further, we presented multiple parameterizations of rotors and motors as well as different cost functions for rotor and motor estimation from noisy point data.

This work is extended in this paper with inspiration from the results of Valkenburg and Dorst [26] on the estimation of motors from sets of heterogeneous objects in the conformal model. We also expand our work on point sets, as presented in [24], by introducing cost functions for motor estimation from 3D line and plane correspondences. Further, we explain how these cost functions make it possible to use other geometric objects like spheres, circles and tangent vectors in the conformal model in the estimation process.The use of automatic differentiation makes it efficient to implement optimization for a wide range of cost functions.

The paper is organized as follows: Sect. 2 introduces geometric algebra and the conformal model. Further, Sect. 3 presents motor estimation from observations of geometric objects in the conformal model. Section 4 presents experimental results. Finally, Sect. 5 concludes the paper.

\section{Geometric Algebra and the Conformal Model}

Geometric algebra is an approach to geometry based on the work of W. Clifford who combined Grassmann's exterior algebra with Hamilton's quaternions, and created what he termed geometric algebra. Hestenes developed geometric algebra further in his books $[11,12]$ and later introduced the conformal model in [15].

The elements of a geometric algebra are called multivectors. The geometric algebra over the 3 -dimensional Euclidean space $\mathbb{R}^{3}$ is denoted $\mathbb{R}_{3}$. The notation $\mathbb{R}_{3}^{r}$ refers to the $r$-grade elements of $\mathbb{R}_{3}$; e.g., $\mathbb{R}_{3}^{2}$ refers to the elements of $\mathbb{R}_{3}$ of grade 2 - the bivectors. The notation $\mathbb{R}_{3}^{+}$refers to the elements of $\mathbb{R}_{3}$ of even grade. The conformal model of geometric algebra is denoted $\mathbb{R}_{4,1}$ with the null basis $\left\{e_{1}, e_{2}, e_{3}, n_{o}, n_{\infty}\right\}$. The basis vector $n_{\infty}=e_{-}-e_{+}$represents the point at infinity and the basis vector $n_{o}=\left(e_{-}+e_{+}\right) / 2$ represents an arbitrary origin. These basis vectors have the properties $n_{\infty}^{2}=n_{o}^{2}=0$ and 
$n_{\infty} \cdot n_{o}=-1$. The notation $e_{i j}$ is shorthand for the outer product $e_{i} \wedge e_{j}$ of the vectors $e_{i}, e_{j} \in \mathbb{R}_{3}^{1}$. The highest grade element of $\mathbb{R}_{3}$, the Euclidean pseudoscalar, is denoted $I_{3}$. The conformal pseudoscalar is denoted $I$. The conformal dual of a multivector $X$ is denoted $X^{*}=X \cdot I^{-1}$. The element of grade $r$ of a multivector $X$ is extracted using the grade projection operator $\langle X\rangle_{r}$.

Vectors $x \in \mathbb{R}_{3}^{1}$ maps to points $p \in \mathbb{R}_{4,1}^{1}$ according to $p=x+\frac{1}{2} x^{2} n_{\infty}+n_{o}$. Lines $\Lambda \in \mathbb{R}_{4,1}^{3}$ are constructed through the outer product of two conformal points and the point at infinity: $\Lambda=p_{1} \wedge p_{2} \wedge n_{\infty}$. Planes $\Pi \in \mathbb{R}_{4,1}^{4}$ are constructed through the outer product of three conformal points and the point at infinity $\Pi=p_{1} \wedge p_{2} \wedge p_{3} \wedge n_{\infty}$. Dual planes are denoted $\pi \in \mathbb{R}_{4,1}^{1}$.

Rigid body motions can be represented in the conformal model using motors

$$
M=T R
$$

where $T=1-\frac{1}{2} t n_{\infty}$ is a translator with translation vector $t \in \mathbb{R}_{3}^{1}$, and $R \in \mathbb{R}_{3}^{+}$is a rotor. A rotor $R$ can be written in the form

$$
R=\cos \left(\frac{\theta}{2}\right)-\sin \left(\frac{\theta}{2}\right) B,
$$

where $\theta \in \mathbb{R}$ is the rotation angle and $B \in \mathbb{R}_{3}^{2}$ is a unit bivector that encodes the rotation plane. It is noted that rotors are isomorphic to the unit quaternions.

Define $\mathbb{M}=\operatorname{span}\left\{1, e_{12}, e_{13}, e_{23}, e_{1} n_{\infty}, e_{2} n_{\infty}, e_{3} n_{\infty}, I_{3} n_{\infty}\right\}$. The motor manifold $\mathcal{M}$ is defined as the set

$$
\mathcal{M}=\{M: M \in \mathbb{M}, M \widetilde{M}=1\} .
$$

The motors can be written as the exponential of a unit bivector $\Lambda^{*}$

$$
M=\exp \left(-\frac{1}{2} \Lambda^{*}\right),
$$

where $\Lambda^{*} \in \operatorname{span}\left\{e_{12}, e_{13}, e_{23}, e_{1} n_{\infty}, e_{2} n_{\infty}, e_{3} n_{\infty}\right\}$. More specifically, $\Lambda^{*}=$ $\theta B+t n_{\infty}$ where $B \in \mathbb{R}_{3}^{2}$ and $t \in \mathbb{R}_{3}^{1}$. It can be shown that $\Lambda^{*}$ is a dual line representing the screw axis of the rigid body motion, see [6]. Following [27], the exponential formulation in (2.4) can be written in terms of the constituent elements of different grades as

$$
\begin{aligned}
& \langle M\rangle_{0}=\cos \left(\frac{\theta}{2}\right) \\
& \langle M\rangle_{2}=\sin \left(\frac{\theta}{2}\right) B+\cos \left(\frac{\theta}{2}\right) t_{\perp} n_{\infty}+\operatorname{sinc}\left(\frac{\theta}{2}\right) t_{\|} n_{\infty} \\
& \langle M\rangle_{4}=\sin \left(\frac{\theta}{2}\right) B t_{\perp} n_{\infty},
\end{aligned}
$$

where $t_{\perp}=(t \wedge B) / B$ is the rejection of $t$ by $B$ and $t_{\|}=(t \cdot B) / B$ is the projection of $t$ in $B$. As described in [7], the rotor $R$ of a motor $M=T R$ can be extracted by

$$
R=-n_{o} \cdot\left(M n_{\infty}\right) .
$$


The translation vector $t \in \mathbb{R}_{3}$ can then be found using

$$
t=-2\left(n_{o} \cdot M\right) / R
$$

The bivector $B$ is found as the normalized grade 2 part of the rotor $R$ :

$$
B=\langle R\rangle_{2} /\left\|\langle R\rangle_{2}\right\| \text {. }
$$

A geometric object $X \in \mathbb{R}_{4,1}$ is displaced by a motor $M \in \mathcal{M}$ using the sandwich product

$$
X^{\prime}=M X \widetilde{M}
$$

where $X^{\prime}$ is the displaced object.

\section{Motor Estimation from Observations of Conformal Objects}

In this section a new approach to motor estimation is proposed based on non-linear least squares optimization of the form

$$
\min _{M \in \mathcal{M}} F(M) \text {. }
$$

where $F: \mathcal{M} \mapsto \mathbb{R}$ is the cost function to be minimized and $M$ is a conformal motor on the motor manifold $\mathcal{M}$. The cost function $F$ can be written

$$
F(M)=\frac{1}{2} \sum_{i=1}^{N}\left(f_{i}(M)\right)^{2}=\frac{1}{2} \sum_{i=1}^{N} \sum_{j=1}^{p}\left(r_{i j}(M)\right)^{2},
$$

where each observation $i$ corresponds to a vector $f_{i}(M)=\left(r_{i 1}, \ldots, r_{i p}\right)^{\top}$ of $p$ residuals $r_{i j} \in \mathbb{R}$. It is seen that the cost function $F$ has $m=N p$ residuals where $N$ is the number of observations. A residual is a scalar measure for the discrepancy between the model and the observed data [16].

It must be ensured that the solution stays on the motor manifold during the optimization. In this paper, this is achieved using the exponential map in (2.5) to (2.7). The use of the exponential map in optimization on manifolds has previously been used in pose estimation on $S E(3)$ [21], and in numerical integration on $S O(3)$ and $S E(3)$ [10]. The motor $M_{k+1} \in \mathcal{M}$ in iteration $k+1$ is calculated as

$$
M_{k+1}=\exp \left(\Lambda^{*}\left(\mathbf{a}_{k+1}\right)\right) M_{k},
$$

where $\mathbf{a}_{k+1} \in \mathbb{R}^{6}$ and

$$
\Lambda^{*}(\mathbf{a})=\sum_{I} a_{I} \Lambda_{I}^{*}, \Lambda_{I}^{*} \in\left\{e_{12}, e_{13}, e_{23}, e_{1} n_{\infty}, e_{2} n_{\infty}, e_{3} n_{\infty}\right\} .
$$

Here, $\mathbf{a}_{k+1}$ is the step calculated using a nonlinear least-squares method [16], e.g., BFGS, nonlinear conjugate gradients or the Levenberg-Marquardt method. The method used in this work is the Levenberg-Marquardt method. This means that $\Lambda^{*}\left(\mathbf{a}_{k+1}\right)$ is in the tangent space of $\mathcal{M}$ at $M_{k}$. Thus, $M_{k+1}$ is on the motor manifold $\mathcal{M}$ and satisfies $M_{k+1} \widetilde{M}_{k+1}=1$ whenever $M_{k} \widetilde{M}_{k}=1$. 


\subsection{Motor Estimation from Points}

Consider a rigid body that is displaced by a motor $M \in \mathcal{M}$. Let $\left\{p_{i}\right\}, p_{i} \in \mathbb{R}_{4,1}^{1}$ be a set of points on the rigid body in the initial configuration, and let $\left\{q_{i}=M p_{i} \widetilde{M}\right\}$ be the set of same points in the displaced configuration. The sets $\left\{p_{i}\right\}$ and $\left\{q_{i}\right\}$ are called point clouds. Motor estimation is to find the motor $M$ given $\left\{p_{i}\right\}$ and $\left\{q_{i}\right\}$.

One possible formulation of this optimization problem employs the inner product between two conformal points

$$
\min _{M \in \mathcal{M}} \frac{1}{2} \sum_{i=1}^{N}\left(M p_{i} \widetilde{M} \cdot q_{i}\right)^{2} .
$$

In this formulation, the measure that is optimized is the squared distance between each two points, resulting in a 1-dimensional residual vector for each observation. This however, is not a good formulation for the cost function as is shown in the experimental results in [24] where the optimization process converge slowly close to the solution.

An alternative solution is to project the points down to the 3dimensional Euclidean model after the transformation by the motor $M$, and then to use the residual errors along each of the coordinate axes, resulting in a 3-dimensional residual vector for each observation

$$
\min _{M \in \mathcal{M}} \frac{1}{2} \sum_{i=1}^{N}\left(\mathrm{P}_{\mathbb{R}_{3}^{\frac{1}{3}}}\left(M p_{i} \widetilde{M}\right)-\mathrm{P}_{\mathbb{R}_{3}^{\frac{1}{3}}}\left(q_{i}\right)\right)^{2} .
$$

In [24], we found that this gave a significantly faster rate of convergence in experiments.

\subsection{Motor Estimation from 3D Line Correspondences}

The next step is to consider motor estimation from two sets of 3D line correspondences. Lines have been widely used in tracking, visual servoing and pose estimation [1], however most of this work is based on optimizing 2D3D line correspondences with error metrics based on image features and not 3D-3D line correspondences. Bartoli and Sturm [1] present two reasons for this. First, there is no global minimal parameterization for lines representing their 4 degrees of freedom with 4 global parameters, and second, there are no universally agreed error metric for comparing lines.

We present three formulations for motor estimation from 3D line correspondences. Formally, given a set of direct or dual line correspondences

$$
\left\{\left(\Lambda_{1_{i}}, \Lambda_{2_{i}}\right)\right\},
$$

the aim is estimate the motor $M$ that transforms the set $\left\{\Lambda_{1_{i}}\right\}$ onto the set $\left\{\Lambda_{2_{i}}\right\}$, that is,

$$
\Lambda_{1_{i}}=M \Lambda_{2_{i}} \widetilde{M} .
$$

The first formulation is based on the "Lin_3D" method in [1], and is based on direct comparison of line coordinates. The optimization problem can then be formulated as 


$$
\min _{M \in \mathcal{M}} \frac{1}{2} \sum_{i=1}^{N}\left(M \Lambda_{2_{i}} \widetilde{M}-\Lambda_{1_{i}}\right)^{2},
$$

resulting in a 6 -dimensional residual vector for each observation.

The other formulation we present is based directly on the geometry of two lines [26]. This formulation is based on the angle and the common normal between two lines. Given two normalized dual lines $\Lambda_{1}^{*}$ and $\Lambda_{2}^{*}$, the common normal Euclidean vector $w \in \mathbb{R}_{3}^{1}$ can be found by computing the motor

$$
\begin{aligned}
M & =T R \\
& =\frac{1}{2} \Lambda_{1}^{*} / \Lambda_{2}^{*} .
\end{aligned}
$$

A measure for the angle between the two lines is found from

$$
\cos \left(\frac{\theta}{2}\right)=\frac{1}{2}\left\langle\Lambda_{1}^{*} / \Lambda_{2}^{*}\right\rangle .
$$

The common normal vector $w$ can then be found as the rejection of the translation vector $t \in \mathbb{R}_{3}^{1}$ of the translator $T$ in the rotation plane bivector $B \in \mathbb{R}_{3}^{2}$ of the rotor $R$.

Given the motor $M$ and the set of normalized dual line correspondences $\left\{\left(\Lambda_{1_{i}}^{*}, \Lambda_{2_{i}}^{*}\right)\right\}$ the residual common normal vector $w \in \mathbb{R}_{3}^{1}$ for line set $i$ can be found from

$$
\frac{1}{2} M \Lambda_{1_{i}}^{*} \widetilde{M} / \Lambda_{2_{i}}^{*}
$$

The optimization problem can then be formulated as

$$
\min _{M \in \mathcal{M}} \frac{1}{2} \sum_{i=1}^{N}\left(w_{i}+(1-\cos (\theta / 2))_{i}\right)^{2},
$$

resulting in a 4-dimensional residual vector for each observation.

A 2-dimensional residual vector for each observation can be formed by using the Euclidean norm of the common normal vector $w,\|w\| \in \mathbb{R}$, and the measure for the angle between the lines.

\subsection{Motor Estimation from Plane Correspondences}

This section presents a new method for motor estimation from correspondences of 3D planes. The proposed method holds for both direct and dual planes. Valkenburg and Dorst [26] employ the inner product between two planes as the similarity measure used in the optimization. For two dual planes $\pi_{1}, \pi_{2} \in \mathbb{R}_{4,1}^{1}$, this results in the measure

$$
\cos (\theta)=\left\langle\pi_{1} \pi_{2}\right\rangle .
$$

However, this measure does not include any information regarding the distance from the origin along the respective normal vectors of each plane. The estimated motor based on this measure will only contain the rotor and not the translator.

In this work we present a formulation that is able to estimate not only the rotor components of the motor, but also the components related to the 
translator. Similarly to creating a motor through the geometric product of two lines, we form a general rotor $G=T R \widetilde{T}$, which is the rotor $R$ translated by the translator $T=1-\frac{1}{2} w n_{\infty}$, through the geometric product of two planes,

$$
G=\frac{1}{2} \pi_{2} / \pi_{1} .
$$

Note that a translator is formed if the unit vectors of the two planes are parallel. The distance measure can then be found by decomposing the general rotor and finding the translation vector $w \in \mathbb{R}_{3}^{1}$ and taking the Euclidean norm

$$
d=\|w\| \in \mathbb{R} .
$$

The optimization problem can then be formulated with the cost function

$$
\min _{M \in \mathcal{M}} \frac{1}{2} \sum_{i=1}^{N}\left(d_{i}+(1-\cos (\theta / 2))_{i}\right)^{2},
$$

resulting in a 2-dimensional residual vector for each observation.

\subsection{Motor Estimation using Heterogeneous Conformal Objects}

In the preceding sections, only homogeneous sets of conformal objects have been used in the motor estimation. Based on the ideas of Valkenburg and Dorst [26], this is now extended to motor estimation using heterogeneous sets of conformal objects that have all been transformed by the same motor, as shown in Fig. 1 for a point, a line and a sphere.

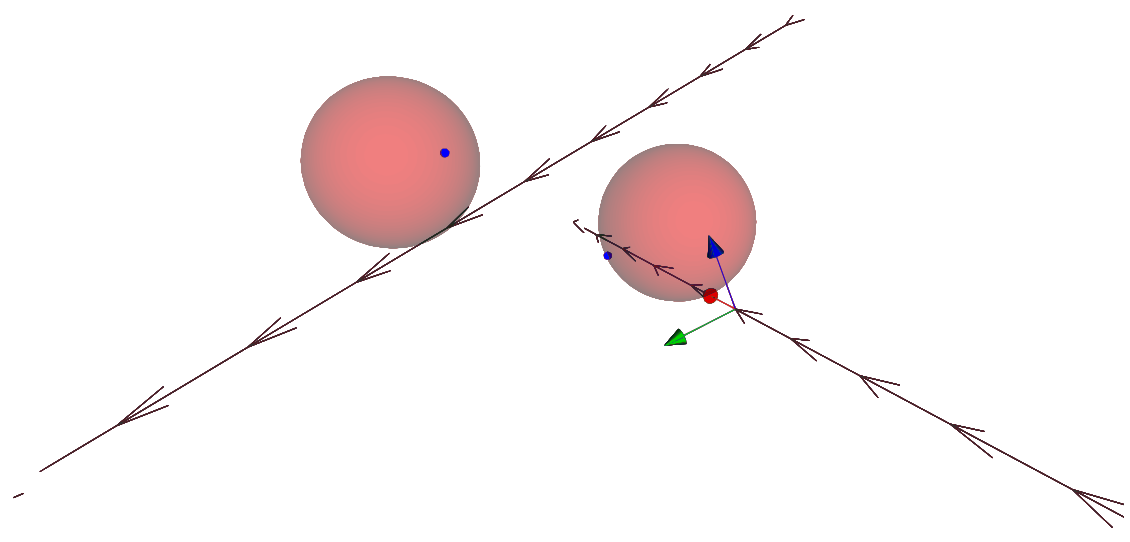

Figure 1. A line, a point and a sphere before and after the transformation by a motor 
In our formulation, this is done with the optimization problem

$$
\begin{aligned}
\min _{M \in \mathcal{M}} & \frac{1}{2} \sum_{i=1}^{N_{1}} w_{1_{i}}\left(\mathrm{P}_{\mathbb{R}_{3}^{1}}\left(M p_{i} \widetilde{M}\right)-\mathrm{P}_{\mathbb{R}_{3}^{1}}\left(q_{i}\right)\right)^{2} \\
+ & \frac{1}{2} \sum_{j=1}^{N_{2}} w_{2_{j}}\left(M \Lambda_{2_{j}} \widetilde{M}-\Lambda_{1_{j}}\right)^{2} \\
+ & \frac{1}{2} \sum_{k=1}^{N_{3}} w_{3_{k}}\left(d_{k}+(1-\cos (\theta / 2))_{k}\right)^{2},
\end{aligned}
$$

where $N_{1}$ are the number of point correspondences, $N_{2}$ are the number of line correspondences, $N_{3}$ are the number of plane correspondences and $w_{1_{i}}, w_{2_{j}}, w_{3_{k}} \in \mathbb{R}$ are weights to adjust the contributions from the different cost functions to the overall solution based on, e.g., the reliability of the data $[26]$.

This formulation can be used to estimate motors from more complex geometric objects as point pairs, circles and tangent vectors. For circles, this can be performed by using the center point and the carrier plane where the center points $c \in \mathbb{R}_{4,1}^{1}$ is extracted using $c=C /\left(-n_{\infty} \cdot C\right)$ and the carrier dual plane is found by $\pi=\left(C \wedge n_{\infty}\right)^{*}$. This can similarly be done for tangent vectors by using the origins of the transformed tangent vectors and the carrier lines. Point pairs can be used by either splitting them into the constituent points or by creating the carrier line.

\subsection{Convexity}

The convexity of motor estimation using non-linear optimization has not been thoroughly investigated in the context of geometric algebra. However, motor estimation is closely related to estimation and control in $S E(3)$, where the analysis can be separated into convergence of translation, and convergence in rotation as given in $S O(3)$. It is known that if deviations in $S O(3)$ are described by the angle $\theta$ and plane of rotation $B$, or with associated metrics like $1-\eta$ or $1-\eta^{2}$ where $\eta=\cos (\phi / 2)$, the convergence to zero deviation can be achieved for all initial conditions except for initial conditions where the solution can get stuck at $|\theta|=\pi$, see $[8,28]$.

The cost functions that we use in the present paper are based on the angle-plane description (2.2) for the deviation in rotation. This is even the case for the cost function in (3.5), which is seen by applying the angle-plane form of the rotor. The step in the Levenberg-Marquart for iteration $k$ is calculated in the tangent space at $M_{k}$ based on the deviation in translation and the deviation in rotation, which is given in terms of the geodesic metric and the plane of rotation in the same tangent space. The update is done with the exponential map and results in a new motor $M_{k+1}$. In this optimization process the incremental convergence properties at each step will be similar to stability properties in the control problem on $S E(3)$. This indicates that the optimization problem will be convex except in the case that the solution gets stuck at $|\theta|=\pi$, which will not be encountered in practice. This agrees with 
the extensive numerical testing that was performed on the method proposed in this paper, with initial angular deviations up to $\bar{\theta}_{0}=\pi$.

\section{Experimental Results}

This section presents experimental results on motor estimation using sets of heterogeneous conformal objects. The experiments were implemented using our framework GAME [23] for multivector estimation, which was presented in [24]. The GAME framework is written in $\mathrm{C}++$ and Python, and is based on the Ceres [20] non-linear optimization framework of Google, and the Versor [4] geometric algebra library of Pablo Colapinto.

We employ the Levenberg-Marquardt (LM) [16] method, where the Jacobian matrices are computed using forward mode automatic differentiation based on dual numbers $[3,13]$. Automatic differentiation computes derivatives with machine precision and works by exploiting the fact that all computer implementations of mathematical functions are composed of a set simple differentiable unary or binary operations. Derivatives of more complex functions are computed by applying the chain rule at each operation and bookkeeping the results [22]. The Jacobian matrices used in this work are of size $N \times 6$, where $N$ is the number of residuals and 6 is the dimension of the tangent space of the motor manifold $\mathcal{M}$ at the motor $M$.

In the following, the ground truth set of homogeneous conformal objects $\left\{X_{i}\right\}, X \in \mathbb{R}_{4,1}, i \in\{1, \ldots, N\}$ are transformed by the motor $M$ to form the set $\left\{X_{i}^{\prime}\right\}$ where $X_{i}^{\prime}=M X_{i} \widetilde{M}$. The motor $M$ which is to be estimated is generated by a rotation of $\pi / 3$ around the $e_{2}$ axis followed by a translation of 1 unit along the $e_{1}, e_{2}$ and $e_{3}$ axes resulting in a motor $M=T R$ with coefficient vector

$$
\mathbf{x}_{M} \approx(0.87,0,-0.5,0,-0.68,-0.43,-0.18,-0.25) .
$$

No noise was added in the experiments and the initial motor $M_{0}=1$ was used in all experiments, if not explicitly stated otherwise. The performance of the different cost functions were evaluated by comparing the coefficients of the estimated motor with the ground truth motor. Two motors are considered to be equal if their coefficient vectors are equal within some tolerance. Given the coefficient vectors $\mathbf{a}, \mathbf{b} \in \mathbb{R}^{8}$, coefficient $i$ of $\mathbf{b}$ is considered to be equal to coefficient $i$ of $\mathbf{a}$ if the following equation holds

$$
\left|a_{i}-b_{i}\right| \leq \alpha+\beta\left|b_{i}\right|,
$$

where $\alpha, \beta \in \mathbb{R}$. In the following experiments we consider two coefficient vectors to be equal if (4.2) holds for

$$
\alpha=1 \times 10^{-8} \quad \text { and } \quad \beta=1 \times 10^{-5} .
$$

The termination criterion of the LM solver is that the relative function tolerance $\epsilon_{F}$ satisfies $\epsilon_{F} \leq 1 \times 10^{-6}$, or that the relative parameter tolerance $\epsilon_{\mathbf{x}}$ satisfies $\epsilon_{\mathbf{x}} \leq 1 \times 10^{-8}$. 


\subsection{Motor Estimation from 3D Point Correspondences}

In [24], we presented motor estimation from sets of noisy point correspondences. Using the LM method we were able to estimate the motor that transformed one set of conformal points to the other set using 1,00,000 observations with a computation time of $0.5040 \mathrm{~s}$.

With no noise added, our method was able to estimate the correct motor from 10 points in 4 iterations within tolerances $\alpha=1 \times 10^{-9}$ and $\beta=1 \times 10^{-5}$.

\subsection{Motor Estimation from 3D Line Correspondences}

Motor estimation using 10 3D line correspondences with three different cost functions was performed. The results are shown in Fig. 2. The cost function in (3.8) with 6 residuals converged in 4 iterations and terminated due to the parameter tolerance $\epsilon_{\mathbf{x}}$, while the cost function in (3.13) with 4 residuals per observation converged in 7 iterations, with termination due to $\epsilon_{F}$. A version of (3.13) using the norm of the common normal between the line correspondences and thus 2 residuals per observation performed slightly worse and converged in 15 iterations, with termination due to $\epsilon_{F}$. The optimization problems based on (3.13) converged quickly during the first iteration while it converged slowly close to the solution, that is, when the distance and angle

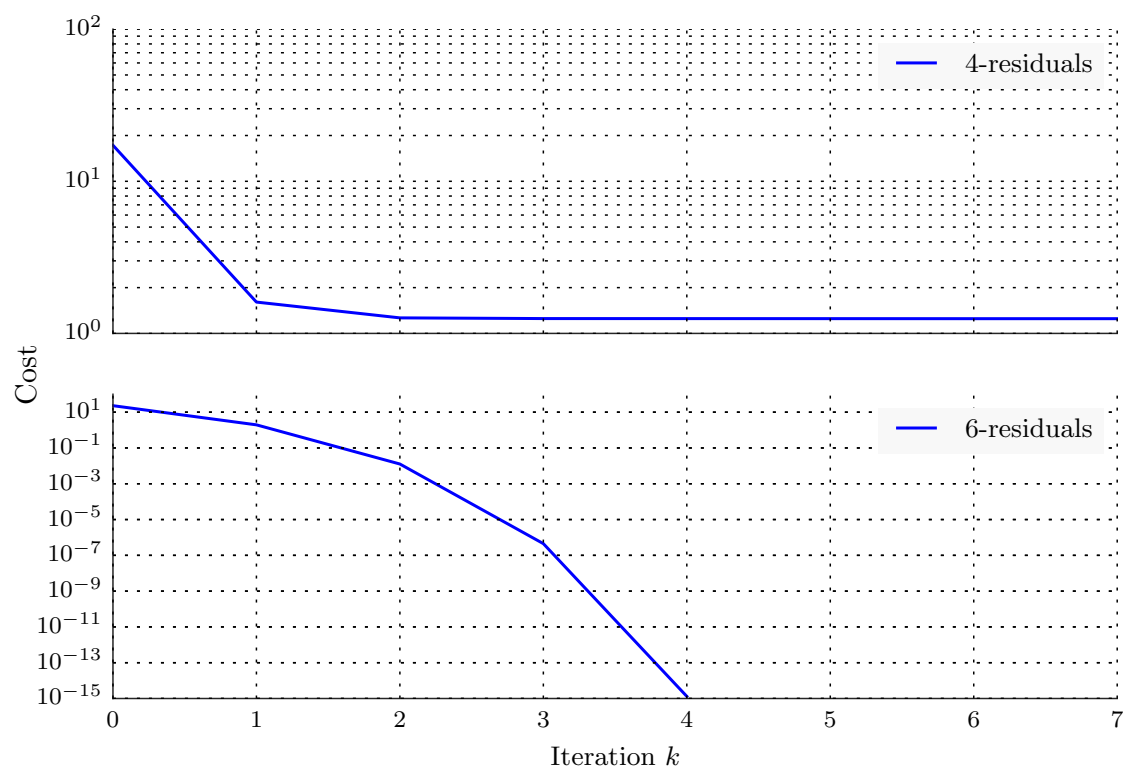

Figure 2. Convergence of the cost function for motor estimation using 3D line correspondences with two different cost functions. The cost function in (3.8) with 6 residuals per observation converged in 4 iterations and terminated due to the parameter tolerance $\epsilon_{\mathbf{x}}$, while the cost function in (3.13) with 4 residuals per observation converged in 7 iterations, with termination due to the function tolerance $\epsilon_{F}$ 


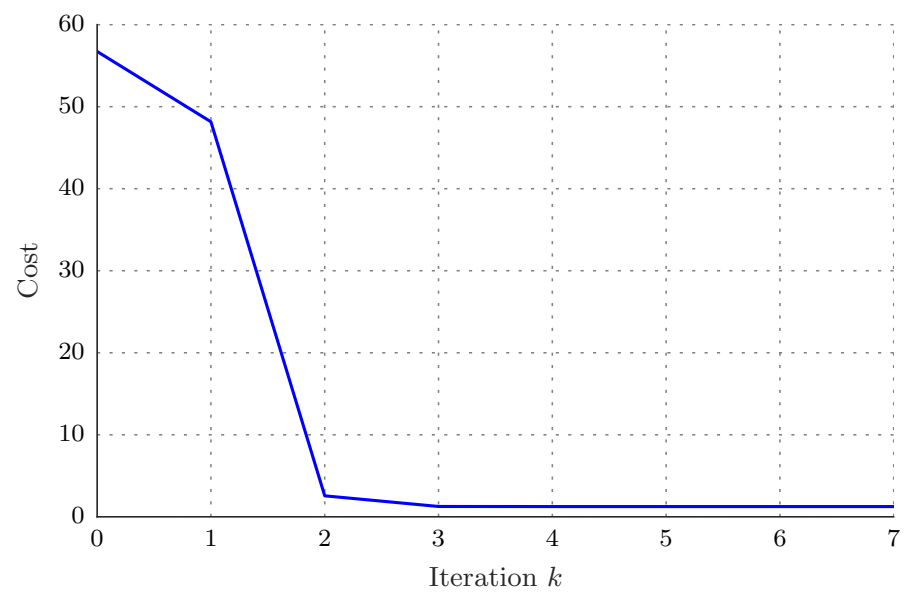

FiguRE 3. Convergence of the cost function for motor estimation using 3D plane correspondences. The cost function in (3.17) with 2 residuals per observation converged in 7 iterations

between the lines are small. The best cost function was the cost function in (3.8) employing 6 residuals per observation. This gave the smallest number of iterations, and it was the only cost function that was able to satisfy the tolerances in (4.3). The two other cost functions were only able to satisfy the condition in (4.2) with $\alpha=1 \times 10^{-3}$. By decomposing the estimated motor, we found that there were errors in both the translational and rotational components with an angle error $\bar{\theta} \approx 3 \times 10^{-4} \mathrm{rad}$ and distance error $\bar{d} \approx$ $1.4 \times 10^{-4} \mathrm{~m}$.

\subsection{Motor Estimation from 3D Plane Correspondences}

Motor estimation using 10 plane correspondences was performed and the results are shown in Fig. 3. Optimization with the cost function in (3.17) with 2 residuals per observation converged in 7 iterations and satisfied the condition in (4.2) with $\alpha=1 \times 10^{-3}$ and $\beta=1 \times 10^{-5}$. The LM solver terminated due to the function tolerance $\epsilon_{F}$.

\subsection{Motor Estimation from Sets of Heterogeneous Conformal Objects}

Motor estimation with the cost function in (3.18) using points, lines and planes gave the results shown in Fig. 4. Without noise, when all objects were transformed by the same motor, we were able to estimate the motor with $\alpha=1 \times 10^{-5}$ in 4 iterations with an initial angular error of $\bar{\theta}_{0}=\pi / 3$. In addition, our method was able to estimate the motor with the same precision with an initial angular error of $\bar{\theta}_{0}=\pi$, demonstrating the robustness of our proposed method. Using the same initial motors and with noise, the optimization converged in 13 and 19 iterations. The noise was applied to the sets $\left\{X_{i}^{\prime}\right\}$ by applying a motor with a small translation and rotation. Different noise motors were applied to different sets, that is, the point set were 


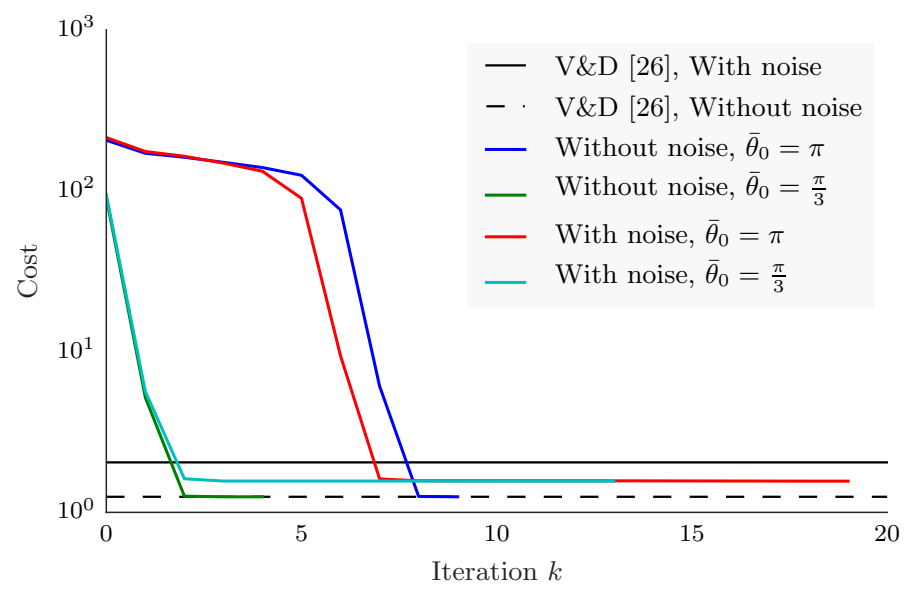

Figure 4. Convergence of the cost function for motor estimation using sets of heterogeneous conformal objects points, lines and planes. Without noise, the motor was estimated with $\alpha=1 \times 10^{-5}$ in 4 iterations with an initial angular error of $\bar{\theta}_{0}=\pi / 3$, and in 9 iteration with $\bar{\theta}_{0}=\pi$. With noise, the initial convergence is comparable, but due to noise the optimization terminated after 13 and 19 iterations. Comparison with the method of Valkenburg and Dorst [26] shows that our method converged with the same accuracy in the noise free case and with improved accuracy when there was noise

transformed by another noise motor than the set of lines and planes. In these experiments, the weights $w_{1_{i}}=w_{2_{j}}=w_{3_{k}}=1$, in (3.18), were used. Our method performs equally well as the method by Valkenburg and Dorst [26] when no noise is present. When noise is present our method performs slightly better.

\section{Conclusion}

In this paper we have presented a novel method for non-linear rigid body motion estimation using point, line and plane correspondences where the rigid body motions were represented using motors in the conformal model of geometric algebra. The presented method makes is possible to estimate motors using both homogeneous and mixed or heterogeneous sets of objects, that is, using observations of points, lines and planes all transformed by the same motor. In addition, these formulations can be used to estimate conformal motors from correspondences of more complex objects, e.g., spheres, circles and tangent vectors. As opposed to the method of Valkenburg and Dorst [26] our method is able to estimate the translational part of the motor using only lines and planes. This is due to the use of the common normal for line correspondences and the translation in the rotation plane for plane 
correspondences. Moreover, our method had slightly better performance than the method of Valkenburg and Dorst in the presence of noise. Our method was very robust in the numerical experiments, and converged consistently with initial angular deviations up to $\pi$.

The Levenberg-Marquardt nonlinear least-squares optimization method was used and Jacobian matrices were computed using automatic differentiation based on dual numbers. The presented method ensures that the solution in each iteration is on the motor manifold by computing the update step in the tangent space and computing the resulting motor using the exponential map.

Open Access. This article is distributed under the terms of the Creative Commons Attribution 4.0 International License (http://creativecommons.org/licenses/ by $/ 4.0 /$ ), which permits unrestricted use, distribution, and reproduction in any medium, provided you give appropriate credit to the original author(s) and the source, provide a link to the Creative Commons license, and indicate if changes were made.

\section{References}

[1] Bartoli, A., Sturm, P.: The 3D line motion matrix and alignment of line reconstructions. Int. J. Comput. Vis. 57(3), 159-178 (2004)

[2] Bayro-Corrochano, E., Daniilidis, K., Sommer, G.: Motor algebra for 3D kinematics: the case of the hand-eye calibration. J. Math. Imaging Vis. 13(2), 79$100(2000)$

[3] Clifford, W.K.: Preliminary Sketch of Biquaternions. Proc. Lond. Math. Soc. 4, 361-395 (1873)

[4] Colapinto, P: (2011) Versor: spatial computing with conformal geometric algebra. Master's thesis, University of California at Santa Barbara. Available at: http://versor.mat.ucsb.edu

[5] Daniilidis, K.: Hand-eye calibration using dual quaternions. Int. J. Robot. Res. 18(3), 286-298 (1999)

[6] Dorst, L.: The representation of rigid body motions in the conformal model of geometric algebra. In: Rosenhahn, B., Klette, R., Metaxas, D. (eds) Human motion: understanding, modelling, capture, and animation, vol. 36 of Computational Imaging and Vision, pp. 507-529. Springer, Netherlands (2008)

[7] Dorst, L., Fontijne, D., Mann, S.: Geometric algebra for computer science: an object-oriented approach to geometry. Morgan Kaufmann Publishers Inc., San Francisco (2007)

[8] Egeland, O., Dalsmo, M., Sørdalen, O.J.: Feedback control of a nonholonomic underwater vehicle with a constant desired configuration. Int. J. Rob. Res. 15(1), 24-35 (1996)

[9] Gebken, C., Perwass, C., Sommer, G.: Parameter estimation from uncertain data in geometric algebra. Adv. Appl. Clifford Algebras. 18(3-4), 647$664(2008)$ 
[10] Hairer, E., Lubich, C., Wanner, G.: Geometric numerical integration : structure-preserving algorithms for ordinary differential equations. Springer series in computational mathematics. Springer, Berlin (2006)

[11] Hestenes, D.: New foundations for classical mechanics, vol. 15 of fundamental theories of physics. Springer, Netherlands (1986)

[12] Hestenes, D., Sobczyk, G.: Clifford algebra to geometric calculus- a unified language for mathematics and physics, vol. 5 of fundamental theories of physics. Springer, Netherlands (1984)

[13] Hoffmann, P.H.W.: A Hitchhiker's guide to automatic differentiation. Num. Algorithms 1-37 (2015)

[14] Lasenby, J., Fitzgerald, W.J., Lasenby, A.N., Doran, C.J.L.: New geometric methods for computer vision: an application to structure and motion estimation. Int. J. Comput. Vis. 26(3), 191-213 (1998)

[15] Li, H., Hestenes, D., Rockwood, A.,: Generalized Homogeneous Coordinates for Computational Geometry. In: Sommer, G. (ed) Geometric computing with Clifford Algebras, pp. 27-59. Springer, Berlin (2001)

[16] Nocedal, J., Wright, S.J,: Numerical optimization. 2 edition, Springer, New York, (2006)

[17] Perwass, C.: Applications of geometric algebra in computer vision-the geometry of multiple view tensors and 3d-reconstruction. $\mathrm{PhD}$ thesis, University of Cambridge (2000)

[18] Rivera-Rovelo, J., Herold-García, S., Bayro-Corrochano, E.: Geometric handeye calibration for an endoscopic neurosurgery system. In: ICRA, pp. 14181423. IEEE (2008)

[19] Rosenhahn, B., Sommer, G.: Pose estimation in conformal geometric algebra part I: the stratification of mathematical spaces. J. Math. Imaging Vis. 22(1), 27-48 (2005)

[20] Sameer, A., Keir, M., et al.: Ceres solver. http://ceres-solver.org

[21] Sarkis, M., Diepold, K.: Camera-pose estimation via projective newton optimization on the manifold. IEEE Trans. Image Process., 21(4), 1729-1741 (2012)

[22] Sommer, H., Pradalier, C., Furgale, P.: Automatic differentiation on differentiable manifolds as a tool for robotics. In: Int. Symp. on Robotics Research (ISRR) (2013)

[23] Tingelstad, L.: GAME-Geometric Algebra Multivector Estimation. Available at: http://github.com/tingelst/game/ (2016)

[24] Tingelstad, L., Egeland, O.: Automatic multivector differentiation and optimization. Submitted to Advances in Applied Clifford Algebras. Available at: http://folk.ntnu.no/tingelst/2016_aaca_tingelstad_egeland.pdf (2016)

[25] Valkenburg, R., Alwesh, N.: Calibration of target positions using conformal geometric algebra. In: Dorst, L., Lasenby, J., (eds.) Guide to geometric algebra in practice, pp. 127-148. Springer, London (2011)

[26] Valkenburg, R., Dorst, L.: Estimating motors from a variety of geometric data in $3 \mathrm{D}$ conformal geometric algebra. In: Dorst, L., Lasenby, J., (eds.) Guide to geometric algebra in practice, pp. 25-45. Springer, London (2011)

[27] Wareham, R., Cameron, J., Lasenby, J.: Applications of conformal geometric algebra in computer vision and graphics. In: Li, H., Olver, P.J., Sommer, G. (eds.) Computer algebra and geometric algebra with applications, pp. 329-349. Springer, Berlin (2005) 
[28] Wen, J.T.Y., Kreutz-Delgado, K.: The attitude control problem. IEEE Trans. Autom. Control, 36(10), 1148-1162 (1991)

Lars Tingelstad, Olav Egeland

Department of Production and Quality Engineering

Faculty of Engineering Science and Technology

NTNU, Norwegian University of Science and Technology

Trondheim, Norway

e-mail: lars.tingelstad@ntnu.no

Olav Egeland

e-mail: olav.egeland@ntnu.no

Received: February 21, 2016.

Accepted: May 30, 2016. 